\title{
Results after transplantation using donor hearts with preexisting coronary artery disease
}

Daniel Marelli, MD
Hillel Laks, MD
Stephen Bresson
Abbas Ardehali, MD
Jessica Bresson, BS
Fardad Esmailian, MD
Mark Plunkett, MD
Jaime Moriguchi, MD
Jon Kobashigawa, MD

From the University of California at Los Angeles Heart Transplant Program, Los Angeles, Calif.

Received for publication Aug 7, 2002; revisions requested Sept 30, 2002; revisions received Nov 26, 2002; accepted for publication Dec 16, 2002.

Address for reprints: Hillel Laks, MD, Division of Cardiothoracic Surgery, UCLA School of Medicine, 10833 Le Conte Ave, 62-182 CHS, Box 951741, Los Angeles, CA 90095-1741 (E-mail: hlaks@ surgery. medsch.ucla.edu).

J Thorac Cardiovasc Surg 2003;126:821-5

Copyright () 2003 by The American Association for Thoracic Surgery

$0022-5223 / 2003 \$ 30.00+0$

doi:10.1016/S0022-5223(03)00213-7
Objective: Cardiac allografts with coronary artery disease may permit a selective expansion of the donor pool. Twenty-two recipients who received donor hearts with mild to moderate coronary artery disease on angiography were reviewed. All donor organs had preserved left ventricle function on echocardiogram.

Methods: The procedure was explained to the patients in detail. All survivors have at least 1 year of follow-up. If the coronary arteries of the donor heart were significantly occluded, then the implanting surgeon performed coronary revascularization. Donors were allocated to patients facing imminent death (group I, $\mathrm{n}=4$ ) or to those who would otherwise not have been transplanted (group II, $n=18$ ). Median recipient age was 57 years old for group I and 68 years old for group II. Median follow-up was 25 months for group I and 44 for group II.

Results: Outcome was evaluated using survival and freedom from graft coronary disease as end points. In group I, 3 of the 4 hearts required revascularization. In group II, 10 of the 18 required revascularization. The majority of the revascularizations were recipient saphenous vein grafts $(84.6 \%)$ to the donor left anterior descending artery (50\%). The 1-month and 2-year actual survivals for group I are 75\% and 50\% and 87.5\% and 81.3 for group II. One patient in group I who was in extremis and 3 in group II died at less than 90 days. Group II early deaths had donor risk factor combinations of coronary artery disease, left ventricular hypertrophy, and long distance. Freedom from new graft coronary artery disease was $100 \%$ at 2 years in group I and $87.5 \%$ in group II.

Conclusions: Selective use of donor hearts with coronary artery disease is acceptable. Early deaths are related to recipient factors as well as associated donor risk factors. Donor hearts with mild or moderate coronary artery disease and preserved function on echocardiogram can be used but may require revascularization with recipient conduit and/or percutaneous transluminal coronary artery angioplasty. Coronary disease in donor hearts requires grading and does not categorically preclude use, particularly in risk-matched recipients.

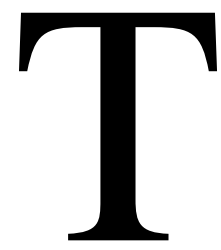

he supply of donor hearts was sufficient to meet recipient demand through the early 1990s. Over the past decade, there has been a considerable increase in the numbers of patients annually listed for cardiac transplantation, which created an undersupply of available organs and extended waiting times significantly. The number of heart transplants performed each year remains in the 2000 to 2500 range while the number of patients listed nationally at year's end increased from 28 
to 56 days for those beginning and ending status I (urgent) and from 221 to 502 days for those beginning and ending status II (nonurgent). ${ }^{1}$ This increased need for donor hearts requires that hearts that were once considered unusable be reevaluated. The use of donor hearts with mild-to-moderate angiogram-diagnosed coronary artery disease (CAD) may permit a selective expansion of the donor pool. Little has been reported on the use of donor hearts with $\mathrm{CAD}^{2-4}$

\section{Methods}

Twenty-two patients received donor hearts with CAD between 1992 and 2001. The patients were divided into 2 groups based on listing at the time of transplant. Group I consisted of United Network for Organ Sharing (UNOS) status I patients $(n=4)$; group II were patients who would otherwise not have been offered a heart transplant $(n=18)$ because of long-term recipient risk (eg, age). For these 18 recipients the donor hearts were otherwise going to be unused; they had been turned down by us and others for standard recipients.

The procedure was explained to the patients in detail, including indications, risks, benefits, possible complications, and other methods of treatment. All patients agreed to accept these donor hearts. If the coronary arteries of the donor heart were significantly occluded, then the implanting surgeon performed coronary revascularization. Another possibility was for the donor heart to undergo percutaneous transluminal coronary artery angioplasty (PTCA) before retrieval.

\section{Group I Recipients}

Group I patients were those who were listed as UNOS status I. Median recipient age for group I was 57 years old (range 44-65). Two recipients were men. Primary indications for transplantation were dilated cardiomyopathy (2), ischemic cardiomyopathy (1), and valvular cardiomyopathy (1).

\section{Group II Recipients}

Group II consisted of those patients who would otherwise not have been offered heart transplants due to associated medical risk (alternate recipients). ${ }^{5}$ The majority of these patients were alternately listed due to old age. Median group II recipient age was 68 years old (range 50.0-74.8). Thirteen recipients were male. Primary indications for transplantation included ischemic cardiomyopathy (75\%), dilated cardiomyopathy (19\%), and retransplant for graft vasculopathy $(6 \%)$.

\section{Definition of Terms}

In the earlier part of this series, any coronary plaque was considered to be evidence of CAD. In the second half of this series (after 1995), less than mild plaque was not considered significant. All patients in this series met the criteria for mild CAD as described below.

Mild CAD: Mild CAD was defined as any discrete stenosis that obstructed greater than $20 \%$ and less than $50 \%$ of the diameter of the artery. Nine of 22 donors were considered mild.

Moderate CAD: Moderate CAD was defined as obstruction that is hemodynamically significant in 1,2 , or 3 vessels $(>50 \%$ diam- eter). If the $\mathrm{CAD}$ was considered moderate, then revascularization was performed. Thirteen of 22 donors were considered to have moderate CAD.

Alternate Recipient: In 1992, the UCLA heart transplant program created a second adult recipient list for patients with heart failure who felt well enough to tolerate surgery. In these patients, long-term prognosis was less certain due to associated medical risk. Such patients were believed to have an acceptable medium-term outlook. This category of patients has become increasingly important, since at this time our heart transplant program had set a recipient age limit of 65 years. An opportunity was created for patients who would typically not receive a transplant because of previous age restriction or other relative medical exclusion criteria to receive hearts that would otherwise not be used.

\section{Organ Preservation and Surgical Technique}

Thyroid hormone $\left(\mathrm{T}_{4}\right)$ infusion is started several hours before retrieval at $0.4 \mu / \mathrm{mL}, 25 \mathrm{~mL} / \mathrm{h}$. Flush-cooling was with University of Wisconsin (UW) solution. Hearts were perfused at a constant pressure of $60 \mathrm{~mm} \mathrm{Hg}$ over a 7-minute period and were transported immersed in hypothermic UW solution $\left(4^{\circ} \mathrm{C}\right)$. Back table coronary bypass grafting has been previously described. ${ }^{4}$ Recipient saphenous vein is used in the majority of cases. Both the distal and proximal anastamoses are performed with the heart immersed in slush for topical cooling before the recipient is started on cardiopulmonary bypass. At the time of implantation, cold electrolyte solution (Plasma-Lyte) solution was infused into the left ventricle to aid in topical cooling and deairing. Reperfusion was carried out with leukocyte-depleted, aspartate/glutamate-enriched, warm blood cardioplegic solution (Buckberg solution) for 3 to 4 minutes, followed by leukocyte-depleted blood for 5 to 10 minutes. Bicaval anastomosis was used for the right atrium. Postoperatively, patients received inhaled nitric oxide if mean pulmonary artery pressure was $>25 \mathrm{~mm} \mathrm{Hg}$ with left atrial pressure $>12 \mathrm{~mm} \mathrm{Hg}$ and cardiac index $<2.2$ to $2.4 \mathrm{~L} / \mathrm{min} / \mathrm{m}^{2}$.

\section{Immunosuppression}

Our heart transplant program has not used induction therapy to complement 3-drug regimens. Methylprednisolone $(7 \mathrm{mg} / \mathrm{kg}$ ) was given at the time of reperfusion and on separation from cardiopulmonary bypass. Cyclosporine (INN: ciclosporin) was administered with the aim of achieving a level of $250 \mathrm{ng} / \mathrm{mL}$. Azathioprine was administered at $2 \mathrm{mg} \cdot \mathrm{kg}^{-1} \cdot \mathrm{d}^{-1}$. Methylprednisolone was first given at a dose of $125 \mathrm{mg}$ every 12 hours for 3 doses. Prednisone was then initiated at $1 \mathrm{mg} \cdot \mathrm{kg}^{-1} \cdot \mathrm{d}^{-1}$ and tapered to $0.1 \mathrm{mg} \cdot \mathrm{kg}^{-1} \cdot \mathrm{d}^{-1}$ over 3 months. For those patients with few rejections, complete weaning from steroids was attempted and achieved in $80 \%$ of cases. If patients had severe, repeated episodes of rejection, then cyclosporine was changed to tacrolimus and azathioprine to mycophenolate mofetil (MMF). More recently, MMF and tacrolimus have been used routinely as part of the initial postoperative 3-drug regimen. All patients received pravastatin postoperatively.

\section{Results}

Donors are summarized in Table 1. All donor hearts ruled out for myocardial infarction by electrocardiogram (ECG), 
TABLE 1. Donor characteristics $(\mathbf{n}=\mathbf{2 2}$ )

\begin{tabular}{lc}
\hline Median age (in years) & $53(40-64)$ \\
Male: female ratio & $17: 5$ \\
Cause of death & \\
Intracranial bleeding & 11 \\
$\quad$ Blunt head trauma & 6 \\
$\quad$ Gunshot wound & 4 \\
Chest trauma & 1 \\
LVEF (\%) & $65(50-75)$ \\
P Mild LVH & 5 \\
Median ischemia time (minutes) & 238 (133-398) \\
\hline LVEF, Left ventricular ejection fraction; $L V H$, left ventricular hypertrophy.
\end{tabular}

creatinine kinase MB fraction (CK-MB) enzymes, and troponin; cardiac echogram did not show left ventricular wall motion abnormalities.

\section{Group I}

Median donor age was 57 years (range 54-58 years). Two of 4 donors were men. Three of the 4 hearts required revascularization. All donors in group I were from distant locations ( $>90$-minute transport time). Median ischemia time was 233.5 minutes (range 133-334 minutes). There was 1 early death at 2 days that was attributed to recipient risk factors (postcardiotomy, ventricular assist device, mechanical ventilation). The 1-month, 1-year, and 2-year actual survivals of group I are $75 \%, 75 \%$, and 50\% (Figure 1).

\section{Group II}

Median donor age was 54 years (range 45-64 years). Fifteen of 18 were men. Ten of the 18 hearts required revascularization. Twelve donors in group II were from distant locations. Median ischemia time was 238 minutes (range 147398 minutes). There were 3 early deaths that were attributed to a combination of CAD, left ventricular hypertrophy, and long distance. The 1-month, 1-year, and 2-year actual survivals are $87.5 \%, 81.3 \%$, and $81.3 \%$ for group II (Figure 1).

\section{Revascularization}

If the coronary arteries of the donor heart were significantly occluded, then the implanting surgeon performed coronary revascularization using recipient conduit. Three of the 4 UNOS status I recipients and 10 of the 18 alternate recipients received hearts that underwent coronary bypass on the back table. Recipient conduits were $84.6 \%$ saphenous vein grafts and $7.7 \%$ left internal thoracic artery pedicle. PTCA was possible in 1 donor heart ( 2 vessels, $7.7 \%$ ). This was carried out by a balloon angioplasty technique only in the donor hospital. These were anatomically favorable lesions (short segment stenosis). Donor targets were 50\% left anterior descending artery, $22.7 \%$ diagonal, $13.6 \%$ obtuse marginal, $9 \%$ posterior descending artery, and $1 \%$ circumflex (Table 2). A total of 26 vessels were revascularized in 13 patients, on average 2 per patient. At 2-year follow-up,

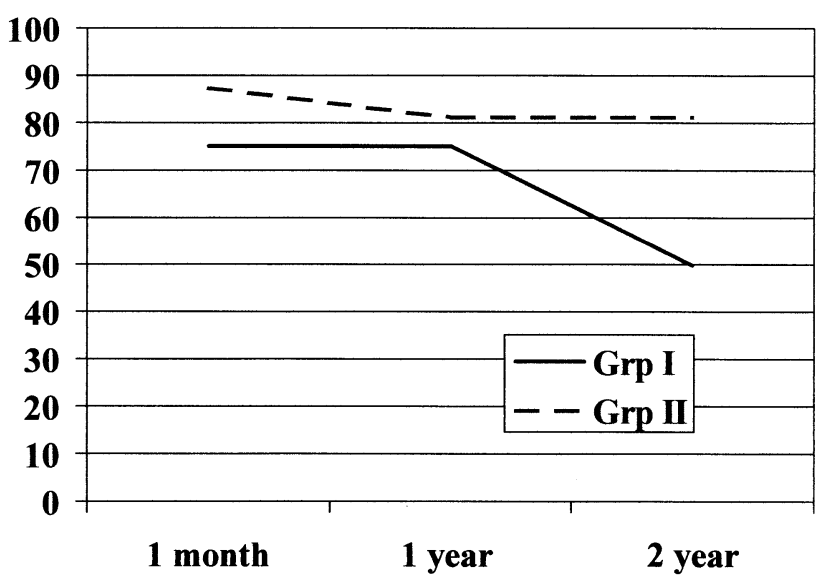

Figure 1. Actual survival for status I recipients (group I) and alternate recipients (group II).

TABLE 2. Revascularization of donor hearts using recipient conduits

\begin{tabular}{lr}
\hline Conduits & 23 \\
Saphenous vein & 2 \\
PTCA & 1 \\
Left internal thoracic artery & 12 \\
Targets & 6 \\
Left anterior descending artery & 3 \\
Diagonal branch & 2 \\
Obtuse marginal branch & 1 \\
Posterior descending artery & \\
Circumflex artery
\end{tabular}

PTCA, Percutaneous transluminal coronary angioplasty.

graft/PTCA patency among all survivors who were revascularized (9/13) was $82 \%$. To date, the longest follow-up is 9 years with 2 of 4 grafts patent. Eight patients have survived more than 5 years. Among current group II survivors, overall graft patency is $47 \%$.

\section{Graft CAD and Rejection}

Freedom from rejection greater than grade $3 \mathrm{~A}$ (grade according to the International Society for Heart and Lung Transplantation) was $100 \%$ at 2 years in group I and $84.6 \%$ in group II. Freedom from new graft CAD and rejection was $100 \%$ at 2 years in group I and $87.5 \%$ in group II.

\section{Discussion}

Donors with CAD represent about 5\% of our experience with nonstandard donors (Appendix 1). The intermediate follow-up results presented in this paper justify continued consideration of such donors in selected recipients. The usefulness of this strategy is unlikely to be applied on a large scale. The gap between donor supply and demand is unlikely to be resolved by any one strategy and is more likely to be tackled by incremental gains. Such an approach 
would likely incorporate all available strategies including lifetime mechanical assistance for older patients with endstage heart failure.

The approach described in the present study was developed because of the need to expand the donor pool. Our first case report of this technique was in the early 1990s when our program had not yet started to use mechanical support to bridge patients to transplant routinely. As well, we found ourselves turning down potential recipients because of an age cutoff of 65 years on our recipient list. This has since been changed to 70 . Patients between the ages of 65 and 70 years old are offered the option of being listed on the alternate list as well as the regular list. This gives them more access to donors as they are preidentified for nonstandard donors in case the organs are unused. The definition of CAD used in this study is arbitrary. At the time (1992) when donors with such a risk factor were first considered, any coronary artery plaque in a donor was generally considered to be a contraindication for acceptance. Our current guidelines shown in Appendix 1 reflect a change in attitude, and we now consider less than mild calcified plaque as defined above standard. The presence of "any" plaque, however, may not be acceptable in much younger adult recipients or adolescents. All donor organs used in group II recipients were turned down by our program and others for regularly listed patients. Only 1 donor organ used in group I did not require revascularization; this organ might possibly be reclassified by some as standard. In the current era, some of the patients in group I would have likely been considered for mechanical support before transplant.

There are several potential concerns regarding the use of donor hearts with CAD. First, we observed that CAD is not uniform. Although diffuse disease clearly precludes organ donation, discrete mild or moderate disease may not. One may consider brain death as a stress such that if subsequent ECG or echocardiogram is favorable, the chance of an older donor having CAD precluding donation is probably low. This type of screening strategy enables efficient selection of older donors for hearts. Additionally, our group II experience, as well as current clinical evidence, indicates that more than mild left ventricular hypertrophy on screening echocardiogram is an important risk factor. ${ }^{6}$

The preservation method used attempted to minimize ischemic injury. There was the possibility that the preservation solution could not penetrate myocardium distal to a coronary artery stenosis. Due to the latter possibility, diffuse 3-vessel CAD likely precludes organ use, although lesser severity may not, particularly if this involves branches of the 3 major coronary arteries with a limited territory at risk. In this regard it is interesting to note that the average number of vessels revascularized was 2 per patient and that 9 of 22 patients did not require revascularization. The use of UW solution has been shown to be potentially advanta- geous. ${ }^{7}$ A leukocyte-depleted reperfusion was used, attempting to minimize reperfusion injury. ${ }^{8}$ We have not attempted to use donor organs with CAD using a different preservation method.

Once the decision was made to accept a donor heart requiring mechanical revascularization, the various choices available were carefully considered. Most commonly, the recipient's saphenous vein was used. This had the advantage of ease of retrieval, no risk of rejection (versus use of donor vein), and back table implantation with the heart completely submerged in ice. Exposure on the back table was ideal for both distal and proximal anastomoses to be constructed. ${ }^{4}$ This also provided the advantage of not adding any additional cardiopulmonary bypass time for the recipient. In patients with ischemic cardiomyopathy who had previous coronary bypass surgery, saphenous vein may not be available. PTCA, particularly with current stent technology, may be ideal as it does not add any ischemic time. Cost and availability in the donor hospital are potential limitations. The pedicled internal thoracic artery may be available in some patients. This graft would be constructed with the donor heart in the recipient's chest during room temperature ischemia, albeit with supplemental topical cooling.

Overall graft/PTCA patency at 2 years was $82 \%$ and current patency among group II patients is $47 \%$. This raises the possibility that some grafts became occluded due to competitive flow from native circulation collaterals as few donor hearts had severe multivessel disease. The need to bypass a vessel was at the discretion of the implanting surgeon, who based the decision on review of the films in the operating room on arrival of the donor heart. On follow-up all of these recipients were labeled as having graft CAD in our database. However, on review of the follow-up catheterizations of this specific cohort, new typical diffuse graft CAD was rarely present. Additionally, we did not observe progression of native disease.

Longer-term studies with larger numbers are needed to separate the patients who received donor hearts with CAD requiring revascularization from those receiving donor organs with CAD not requiring intervention. On the basis of the data in the present study, we can recommend that donor hearts with less than mild or mild plaque in 1 or 2 vessels be considered for most older and middle-aged recipients who are listed as status I. Younger recipients similarly listed would generally be considered better assist device candidates if their condition deteriorates. Donor hearts with more than mild plaque or discrete stenoses in fewer than 3 major territories are similarly considered for status IA patients and older recipients who are status IB or who are on the alternate recipient list. Decisions must be individualized on the basis of donor/recipient size match, risk of elevated pulmonary artery pressures, and anticipated total ischemic time. Presence of left ventricular hypertrophy generally precludes 
use of donor hearts with CAD, particularly if ECG criteria are present indicating long-standing disease. ${ }^{6}$ The intermediate results presented demonstrate proof of the concept of accepting donors with CAD. Previous studies indicate that graft $\mathrm{CAD}$ is multifactorial and that plaque in the donor heart may not progress to graft $\mathrm{CAD} .{ }^{9}$ The use of pravastatin and recipient conduit may account for the satisfactory midterm results observed. ${ }^{10}$

\section{References}

1. http://www.unos.org.

2. Thomson DJ, Kostuk W, Pfluelder P, et al. De novo coronary artery grafting in a heart transplant recipient. J Heart Transplant. 1988;7: 468-70.

3. Schuler S, Matschke K, Loebe M, et al. Coronary artery disease in patients with older donor hearts-morphological features and therapeutic implications. J Heart Lung Transplant. 1992;11:192.

4. Laks H, Gates RN, Ardehali A, et al. Orthotopic heart transplantation and concurrent coronary bypass. J Heart Lung Transplant. 1993;12: 810-5.

5. Laks H, Marelli D. The alternate recipient list for heart transplantation: a model for expansion of the donor pool. Adv Card Surg. 1999;11: 233-44.

6. Marelli D, Laks H, Fazio D, et al. Is the use of donor hearts with left ventricular hypertrophy acceptable? J Heart Lung Transplant. 2000; 19:496-503.

7. Stein D, Drinkwater D, Laks H, et al. Cardiac preservation in patients undergoing transplantation: a clinical trial comparing University of Wisconsin solution and Stanford solution. J Thorac Cardiovasc Surg. 1991;102:657-65.

8. Pearl JM, Drinkwater DC, Laks H, Capouya ER, Gates RN. Leukocyte-depleted reperfusion of transplanted human hearts: a randomized, double-blind clinical trial. J Heart Lung Transplant. 1999; 11:1082-92.

9. Wong CK, Ganz P, Miller L, et al. Role of vascular remodeling in the pathogenesis of early transplant coronary artery disease: a multicenter prospective intravascular ultrasound study. J Heart Lung Transplant. 2001;20:385-92.

10. Kobashigawa JA, Katznelson S, Laks H, et al. Effect of pravastatin on outcomes after cardiac transplantation. N Engl J Med. 1995;333: $621-7$.

\section{Appendix 1. UCLA Nonstandard Donor Guidelines (relative proportions 1992-2001, $n=288$ )}

\begin{tabular}{l} 
Risk Factor \\
\hline Cardiac \\
CAD (5\%) \\
Age $>45$ years, coronary an \\
LVH by ECG criteria ( $2 \%)$ \\
LVH $>$ mild by echocardiogra \\
Low LVEF (9\%) \\
High-dose inotropic requirement \\
Suspected myocardial contus \\
trauma (21\%) \\
Recent cardiac arrest (15\%) \\
Reused heart (1\%) \\
Noncardiac \\
Older age $(17 \%)$ \\
Hepatitis B positive $(1 \%)$ \\
Hepatitis C positive (11\%)
\end{tabular}

$C A D$, Coronary artery disease; $L V H$, left ventricular hypertrophy; $E C G$, electrocardiogram; $L V E F$, left ventricular ejection fraction; $R V$, right ventricle; $C K-M B$, creatine kinase MB isoenzyme; $l g$, immunoglobulin; $H C V$, hepatitis $C$ virus.
Any coronary artery stenosis evident on coronary angiogram or $\geq$ than mild calcified plaque

Normal function on echocardiogram

Abnormal QRS in $\mathrm{V}_{5}$ and $\mathrm{V}_{6}$

Posterior wall thickness $\geq 14 \mathrm{~mm}$

$<50 \%$ on echocardiogram

One inotrope at maximal dose or two inotropes at greater than half maximal dose (dopamine and dobutamine maximal dose $\left.=20 \mu \mathrm{g} \cdot \mathrm{kg}^{-1} \cdot \mathrm{min}^{-1}\right)$

Significant anterior blunt chest injury with RV or septal wall motion abnormality on echocardiogram complemented by elevated CK-MB

Organ retrieved less than 24 hours after cardiac arrest

Age $>55$ years

IgG core antibody positive; IgM unknown

Positive anti-HCV 